\title{
A SOSEKI-based coordinate system interprets global polarity cues in Arabidopsis
}

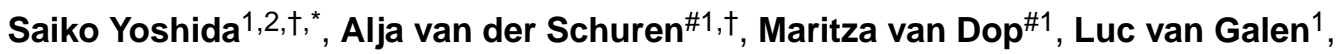 \\ Shunsuke Saiga ${ }^{1, \dagger}$, Milad Adibi $^{3}$, Barbara Möller ${ }^{1}, \dagger$, Colette A. ten Hove ${ }^{1}$, Peter Marhavy ${ }^{2, \dagger}$, \\ Richard Smith $^{3}$, Jiri Friml ${ }^{2}$, and Dolf Weijers ${ }^{1,}$
}

${ }^{1}$ Laboratory of Biochemistry, Wageningen University, Stippeneng 4, Wageningen, the Netherlands ${ }^{2}$ Institute of Science and Technology, Klosterneuburg, Austria ${ }^{3}$ Department of Comparative Development and Genetics, Max Planck Institute for Plant Breeding Research, Cologne, Germany

\# These authors contributed equally to this work.

\section{Abstract}

Multicellular development requires coordinated cell polarization relative to body axes, and translation to oriented cell division1-3. In plants, it is unknown how cell polarities are connected to organismal axes and translated to division. Here, we identify Arabidopsis SOSEKI (SOK) proteins that integrate apical-basal and radial organismal axes to localize to polar cell edges. Localization does not depend on tissue context, requires cell wall integrity and is defined by a transferrable, protein-specific motif. A Domain of Unknown Function in SOK proteins resembles the DIX oligomerization domain in the animal Dishevelled polarity regulator. The DIX-like domain self-interacts and is required for edge localization and for influencing division orientation, together with a second domain that defines the polar membrane domain. Our work shows that SOK proteins locally interpret global polarity cues and can influence cell division orientation. Furthermore, this work reveals that despite fundamental differences, cell polarity mechanisms in plants and animals converge upon a similar protein domain.

\footnotetext{
Users may view, print, copy, and download text and data-mine the content in such documents, for the purposes of academic research, subject always to the full Conditions of use:http://www.nature.com/authors/editorial_policies/license.html\#terms

*Correspondence to: yoshida@mpipz.mpg.de or dolf.weijers@wur.nl.

†Present addresses: Department of Comparative Development and Genetics, Max Planck Institute for Plant Breeding Research, Cologne, Germany (S.Y.); Department of Plant Molecular Biology, University of Lausanne, Lausanne, Switzerland (A.v.d.S.; P.M.); Department of Plant and Microbial Biology, University of Zürich, Zürich, Switzerland (S.S.); VIB-UGent Center for Plant Systems Biology, Ghent, Belgium (B.M.).

Author contributions: D.W. conceived the study. S.Y. performed most of the experiments under the supervision of D.W., and with help from A.v.d.S., L.v.G. and S.S.. M.v.D. Performed BiFC and FRET-FLIM experiments, SOK1 localization in ground tissue and in shr and scr mutants (together with C.A.t.H.), and SOK1/SOK5 swaps. B.M. initiated the project and identified the $S O K 1$ gene. M.A. and R.S.S. performed 3D SOK1 localization analysis. P.M. helped to perform live imaging under J.F.'s supervision. S.Y. and D.W. wrote the manuscript with input from all authors.

Competing interests: Authors declare no competing interests

Data availability: The data that support the findings of this study are available from the corresponding authors upon reasonable request.
} 
Development of multicellular organisms relies on the ability to organize cell differentiation and division relative to body axes and to other cells within the same tissue. Individual cells are polarized, and polarity information is relayed to trigger local outgrowth 1,2 or steer division orientation 1,3. Mechanisms underlying polarization and division orientation are relatively well-understood in yeast and animals4,5. In plants, oriented division is critical for normal development6,7, and several polarly localized proteins have been identified8-15. However, yeast and animal polarity regulators seem to be missing from plant genomes, and it is thought that polarity components and mechanisms are distinct in plant and animal kingdoms16. Components of such mechanisms are elusive. The plant signaling molecule auxin regulates pattern formation, and defects in auxin response often manifest as changes in growth direction or cell division plane6,17. Mutations in the Arabidopsis MONOPTEROS/ AUXIN RESPONSE FACTOR5 (MP) transcription factor 18 cause alterations in division planes in the early embryo19. We argued that mediators of MP function in controlling cell division orientation should be among its transcriptional targets. Starting from a set of MPdependent genes 20 , we here identify a family of novel, polarly localized proteins that link organismal axes to cell polarity and division orientation.

We previously performed transcriptome analysis on globular-stage embryos in which MP activity was locally inhibited20, and identified TMO721 as the most strongly downregulated gene20. The second most strongly down-regulated gene (7.5-fold) is a gene of unknown function, containing a Domain of Unknown Function 966 (DUF966) and named SOSEKI1 (explained below; SOK1; At1g05577). SOK1 has 4 paralogues in the Arabidopsis genome: SOK2 (At5g10150), SOK3 (At2g28150), SOK4 (At3g46110) and SOK5 (At5g59790) (Fig. 1a; Extended Fig. 1) of which SOK5 was also 2.4-fold down-regulated in embryos with reduced MP function.

To determine gene expression domains, nuclear-localized triple GFP (n3GFP) was driven by each $S O K$ promoter, and this revealed that all $S O K$ genes are expressed during embryogenesis and in primary/lateral roots (Extended Fig. 2). To observe protein localization, C-terminal YFP translational fusions were generated. All SOK-YFP patterns mirror pSOK-n3GFP patterns (Fig. 1, 2; Extended Fig. 2). Strikingly, each SOK protein marked novel cellular domains. SOK1-YFP marks the outer/apical edge of young vascular cells, including the pericycle, and in the columella root cap in the primary root (Fig. 1b,c,h). During embryogenesis, SOK1-YFP is first detected in the apical side of lower tier inner cells at the early globular stage (Fig. 2a). Subsequently, SOK1 localizes to the outer apical corner or outer lateral side of vascular cells and outer corners of the hypophysis at transition to heart stage (Fig. 2f; Extended Fig. 3). This pattern is maintained in the post-embryonic root (Fig. 1), and the lateral root (Fig. 2k; Extended Fig. 4). The same localization pattern was observed for SOK1-tdTomato (Extended Fig. 2), and is hence independent of the protein tag. The protein was named SOSEKI1 (Japanese for "cornerstone") for this unique corner localization pattern. The SOK1 protein is highly unstable: SOK1-YFP signal disappears during cell division but is afterwards quickly re-established in lateral root primordia (Fig. 2p) and primary root (Fig. 2q). Treatment of SOK1-YFP roots with the translation inhibitor Cycloheximide (CHX) confirmed protein instability (Extended Fig. 6). 
All other SOK proteins were localized to different subcellular edges. SOK2-YFP localized to the inner basal edge of endodermal cells in the primary (Fig. 1d,i) and lateral root (Fig. 21; Extended Fig. 4), starting in the globular embryo (Fig. 2b; Extended Fig. 3). SOK3-YFP accumulated at the basal side and all corners of most cells in the primary root (Fig. 1e,j), lateral roots (Fig. 2m; Extended Fig. 4) and embryos (Extended Fig. 3). Only faint SOK4YFP accumulation was observed few vascular cells in the primary root (Fig. 1f,k), while it was expressed in lateral root (Fig. 2n; Extended Fig. 4) and embryo (Fig. 2d,i; Extended Fig. 3). SOK5-YFP resembles SOK2 localization in endodermis, QC and lateral root cap in primary root (Fig. 1g,l), yet SOK2 and SOK5 patterns differ during embryogenesis (Fig. 2e,j; Extended Fig. 3). Thus, all SOK proteins are expressed in specific tissues during root initiation, growth and branching, and mark unique polar subcellular domains.

To test whether differences in polar localization among SOK proteins are caused by cell types or protein determinants, we misexpressed SOK1-YFP or SOK2-YFP from the RPS5A promoter that drives broad expression in meristems22. Apical SOK1 polarity was found in all cell types, but misexpressed SOK1 localizes to inner rather than outer edges in cortex and epidermis (Fig. 3a-c). Ectopic SOK2-YFP localizes to inner basal edges of all cells (Fig. 3e). Thus apical/basal polarity is maintained in misexpression lines and appears intrinsic to SOK1 and SOK2 proteins. Strikingly, in these lines, polar localization was found across the embryonic shoot/root axis (Fig. 3d,f), suggesting the existence of a common polarity reference in the entire body. During lateral root initiation however, localization followed the new organ axis (Fig. 2k,1; Extended Fig. 4), suggesting that the SOK-based coordinate system is autonomous to lateral organs. In contrast to apical/basal polarity, inner/outer polarity of SOK1 depended on position relative to the endodermis (Fig. 3a); SOK1 always localized pointing towards the endodermis. Localization in the shortroot (shr) and scarecrow (scr) mutants with impaired endodermal identity23,24 caused loss of edge localization and led to apical accumulation in the mutant cell file (Fig. 3k,l). This suggests that edge localization integrates genetically separable apical-basal and outer-inner axes. The cortexendodermis junction serves as a potent cue for SOK1 localization, which is confirmed by ground tissue-specific expression of SOK1-YFP using the N9135 GAL4 driver line25. In the shared initial for cortex/endodermis, SOK1-YFP is apical, while the protein localized at both opposing edges toward the junction after the periclinal division separating separates endodermis and cortex (Fig. 3m,n). These observations revealed that plant cells and tissues possess a universal coordinate system with an internal reference that is read and integrated by SOK proteins. While other proteins, such as BOR1 can use this internal reference system26,27, it is unclear if other proteins can likewise integrate axes.

Polar localization of plant proteins generally depends on dynamic processes such as membrane trafficking, cytoskeletal dynamics or protein degradation8-10,12,14. In stark contrast, none of 20 tested hormone or drugs affecting these processes, nor changes in light, temperature or gravity angle affected the edge localization of SOK1, SOK2 or SOK3 (Extended Fig. 6; Extended Table 1). This suggests that polar localization follows a pathway that is different from well-known polar proteins such as PINs, BOR1, NIP5 and PEN3 8$10,12,14$. We next tested if the cell wall, or mechanical properties influence SOK localization and found that a brief ( $<20$ minutes) treatment with cell wall-degrading enzymes or high osmotic mannitol solution led to internalization and mis-localization of SOK 
proteins (Fig. 3o-q; Extended Fig. 5). This indicates that cell wall integrity is critical for edge localization and membrane association of SOK proteins.

SOK1-YFP misexpression induced frequent alterations in cell division orientation; in embryos, such divisions were found in all cell types (Fig. 3i,j; Extended Fig. 7), while in roots, defects were strongest in the cortex and epidermis (Fig. 3a,g,h). While root cells normally divide anticlinal to the growth axis (Fig. 3g), misexpression lines displayed either oblique or periclinal divisions generating additional cell layers (Fig. 3h). The same defect was induced by misexpressing non-tagged SOK1, and was accompanied by slightly inhibited root growth (Extended Fig. 7). We utilized this activity in redirecting cell division planes to determine protein determinants and properties required for activity. SOK proteins do not have a signal peptide or predicted transmembrane helices (Extended Fig. 1) and are likely peripherally membrane-associated. To target SOK1 uniformly to the plasma membrane, we fused an N-terminal Myristoylation (Myr) motif 28 to SOK1-YFP. Both polarity and activity (as judged by oblique cell divisions) were strongly diminished in MyrSOK1-YFP roots (Fig. 3r). In contrast, when the same Myr motif was fused to the Cterminus of SOK1-YFP as a negative control, neither polarity nor activity was affected (Fig. $3 \mathrm{~s})$. Thus, insertion in the plasma membrane prevents polar localization, which is in turn required for SOK1 function.

To locate polarity determinants, we generated a series of $\mathrm{N}$ - or C-terminal deletions (Fig. 4ae; Extended Fig. 8) and misexpressed each as a YFP fusion. Deletions $\Delta \mathrm{A}, \Delta \mathrm{B}, \Delta \mathrm{C}$ and $\Delta \mathrm{D}$ caused SOK1 to be localized in the cytosol. Interestingly, $\Delta \mathrm{E}$ localized to the apical edge and induced oblique divisions (Fig. 4c), suggesting that the fragment contained in the $\Delta \mathrm{D}$ $\Delta \mathrm{E}$ segment is sufficient for polar localization and for altering division orientation. Deletions $\Delta \mathrm{F}, \Delta \mathrm{G}, \Delta \mathrm{H}$ and $\Delta \mathrm{I}$ are broadly localized to plasma membrane, suggesting that the $\mathrm{N}$ terminus is not required for localized membrane association per se, but rather for focusing to the edge. Deletions $\Delta \mathrm{J}, \Delta \mathrm{K}$ and $\Delta \mathrm{L}$ were all localized to the cytosol, suggesting that the $\Delta \mathrm{D}$ $\Delta \mathrm{E}$ segment can only direct edge localization if the $\mathrm{N}$-terminus is present. Thus, SOK1 carries two function domains: one for membrane association (middle), and another (Nterminal) for focused polar localization. Both are required for activity in changing division orientation.

To test whether different SOK proteins use similar domains for localization, we replaced successive 50-70 amino acids of the basally localized SOK2-YFP by the corresponding region of SOK1 (Named S2S1A-F; Fig. 4f-j; Extended Fig. 8). While most chimaeras localized to the basal edge (Fig. 4g,j; Extended Fig. 8), S2S1D shifted to the apical edge (Fig. 4h; Extended Fig. 8), similar to SOK1-YFP. The S1S2E showed a mixture of SOK1 and SOK2 localization, at the inner lateral membrane (Fig. 4i; Extended Fig. 8). Thus, polar localization can be transferred between SOK1 and SOK2 using a discrete domain that overlaps with the $\Delta \mathrm{D}-\Delta \mathrm{E}$ "polarity domain" defined by deletions. SOK1 misexpression induces oblique divisions (Fig. 3a), while SOK2 misexpression does not (Fig. 3e). Even when relocated to the SOK1 polarity domain, SOK2 can not alter division orientation (Fig. 4h). Thus, SOK proteins differ from one another not only in their polar localizations, but also in their ability to modify cell division orientation. We finally confirmed that the same domain conferred polarity changes in swaps between SOK1 and SOK5 (Extended Fig. 9). 
This identifies a minimal domain for polar targeting of multiple SOK members. This minimal polarity domain acts in conjunction with the conserved $\mathrm{N}$-terminal domain to direct focused, polar membrane localization to specific cell edges.

SOK proteins are plant-specific, and the function of DUF966 domain is unknown. We used structural homology modeling to identify potential homologues, and found that, despite very low sequence conservation, a part of the DUF966 domain resembles the DIX (Dishevelled/ Axin) domain (Fig. 4k) that is found in a number of animal proteins29,30. The DIX domain mediates head-to-tail oligomerization 31 and is required for clustering of the polarity regulator Dishevelled in Drosophila30,31. Strikingly, the DIX domain is found only in a small number of metazoan proteins, each of which is involved in Wnt signaling and/or planar cell polarity 29,30 , which suggests a convergence of polarity mechanisms across kingdoms. We tested the ability of the DIX-like domain in SOK1 to self-associate. Indeed, Bimolecular Fluorescence Complementation in tobacco leaves (Fig. 41-n) and a FRET assay in Arabidopsis protoplasts (Fig. 4o-q) showed that the predicted DIX domain homodimerizes. The DIX-like domain corresponds to the $\mathrm{N}$-terminal region that is required for edge localization and biological activity (Fig. 4a,d; Extended Fig. 1). Thus, the animal cell polarity protein Dishevelled and SOSEKI1 may use a homologous protein domain for their asymmetric localization and polarity-related function.

Our study identified a novel plant-specific family of polar, edge-localized proteins. Localization to previously unidentified polar domains is important for the activity of SOK1 in influencing the cell division axis. Whether SOK1 and other family members mediate this function during normal development, and what cellular mechanism underlies such activity is yet to be determined, in part through analyzing loss-of-function mutants. Single mutants in individual $S O K$ genes did not so far reveal phenotypes (not shown), but redundancy is likely. Thus, current efforts are aimed at generating complete knockout of all family members. Nevertheless, our analysis of SOK localization identified a universal coordinate system in plants that extends well beyond the global polarity field in the leaf32, and encompasses the entire body axis. SOK proteins that can locally interpret and integrate the global coordinates. Consistent with being the output of a coordinate system, SOK localization is robust, yet seems to rely on cell wall integrity and/or mechanical properties. Further exploration of the mechanisms of SOK localization and function may help revealing the fundamental principles of cell and organismal polarity in plants. This may herald surprising analogies to animal polarity mechanisms, given the adoption of a homologous protein domain in polar proteins across kingdoms.

\section{Methods}

\section{Plant material}

Arabidopsis ecotype Columbia- 0 was used in all experiments except when noted otherwise. The N9135-GAL4 enhancer trap line33 was generated by Jim Haseloff (University of Cambridge, UK) and obtained though the Arabidopsis Biological Resource Centre (ABRC). $s h r-2$ and $s c r-4$ mutants were previously described 34,35 . 
All seeds were sterilized, sown on MS medium with $1 \%$ sucrose and $0.8 \%$ Daishin agar (Duchefa) and vernalized for one day. Plants were grown on soil at $22^{\circ} \mathrm{C}$ under the long-day condition.

\section{Molecular cloning}

All cloning was performed using previously described Ligation-Independent Cloning methods and vectors36.For promoter-GFP lines, $1.2-5 \mathrm{~kb}$ fragments upstream of the ATG of $S O K$ genes were amplified and introduced into upstream of SV40-n3eGFP in pPLV04. For C-terminal translational fusion lines, genomic fragments, including 1.2-5 kb upstream of SOK genes, were introduced into pPLV16 (sYPF2) or pPLV22 (tdTomato). For misexpression lines, $S O K$ cDNAs were amplified, fused with sYFP2 and introduced into pPLV28 downstream of the RPS5A promoter. For gene swap experiments, cDNA fragments of SOK1, SOK2, SOK5 and YFP were amplified, fused by overlap extension PCR and introduced downstream of the RPS5A promoter in pPLV28. For deletion experiments, cDNA fragments from SOK1-YFP were amplified and introduced downstream of the RPS5A promoter in pPLV28. For myristoylated SOK1 constructs, sequences for myristoylation (GGCFSKK) 28 was added to either C-terminus or N-terminus of SOK1 cDNA sequences, and introduced downstream of the RPS5A promoter in pPLV28. The $U A S:$ :SOK1-YFP construct was generated by amplifying SOK1-YFP from the pRPS5A::SOK1-YFP plasmid and introducing it downstream of the GAL4-dependent $U A S$ promoter 37 in pPLV32. The construct was transformed directly into the N9135 enhancer trap line33. For BiFC, the DIX cDNA sequence was amplified from the pRPS5A::SOK1YFP plasmid and cloned into a modified pGreenII vector containing p35S::LIC-n/cYFP. FLIM vectors were generated by cloning the DIX cDNA into pMON 35S::LIC-sYFP or pMON 35S::LIC-sCFP3a.

All constructs were sequenced, and transformed into wild-type Columbia or N9135 GAL4 by floral dip using Agrobacterium strain GV3101(pSoup). All primers used for cloning are listed in the Extrended Table 2.

\section{Microscopic analysis}

Roots were stained by propidium iodide (PI; Sigma-Aldrich) at final concentration of $10 \mu \mathrm{g} / \mathrm{ml}$. Embryos were fixed and stained by $2.2 \%$ Renaissance RS2200 (Renaissance Chemicals Ltd) in PBS buffer at pH 6.9 containing 4\% paraformaldehyde, 5\% glycerol, $4.2 \%$ dimethyl sulfoxide (DMSO) or stained by PI after fixation as previously described6,38.

Confocal imaging was performed with Zeiss LSM700/800 (observation of lateral root) or Leica SP5/II (observation of primary root and embryo) as previously described38. Live imaging was performed with LSM700 as described before39,40. For the 3D imaging of pSOK1-SOK1-YFP and pSOK1-GFP root, Zeiss LSM780 with 2 photon laser (960-990nm) and 500-550nm/575-610nm band-pass filter were used. Analysis of confocal images (3D reconstruction, 3D segmentation) was performed by MorphoGraphX 41. 


\section{Chemical treatments}

All chemicals used to treat SOK-YFP roots are shown in Extended Table 1. Concentration for each chemical was obtained from literature. Seedlings were placed on MS plates containing each chemicals for the durations mentioned in the text and were subsequently imaged by confocal microscopy. As controls for the chemical treatments, the same percentage of DMSO or ethanol used for each chemical treatment were used and no effect was observed. For most of the chemical treatments, final concentrations of DMSO were $<0.5 \%(\mathrm{v} / \mathrm{v})$ and ethanol were $<0.1 \%(\mathrm{v} / \mathrm{v})$.

Plasmolysis was performed either on MS medium with mannitol (Sigma, M9546) at final concentration $0.4 \mathrm{M}$ or by dipping roots in $0.4 \mathrm{M}$ mannitol solution in milliQ water, and stained by FM4-64 at least for $2 \mathrm{~min}$. For cell wall digestion, either 1\% Cellulose R10 (Yakult Honsha Co. L.T.D, Japan) and/or 0.2\% Macerozyme (Duchefa) were used. Cellulose and/or Macerozyme was dissolved in a solution containing $0.4 \mathrm{M}$ mannitol, $10 \mathrm{mM} \mathrm{CaCl}$, $20 \mathrm{mM} \mathrm{KCl}$ and $20 \mathrm{mM}$ MES (pH 5.7).

\section{BiFC and FRET-FLIM}

BiFC was performed as follows: Agrobacterium containing BiFC plasmids were grown overnight in $5 \mathrm{ml} \mathrm{LB}+20 \mathrm{mg} / \mathrm{L}$ Gentamycin, $50 \mathrm{mg} / \mathrm{L}$ kanamycin, $25 \mathrm{mg} / \mathrm{L}$ rifampicin and $2 \mathrm{mg} / \mathrm{L}$ tetracyclin. Cultures were spun down at $4000 \mathrm{rpm}$ for 10 minutes and the bacterial pellet was resuspended in $1 \mathrm{~mL}$ MMAi (5g/L MS salts without vitamins, $2 \mathrm{~g} / \mathrm{L}$ MES, 20g/L sucrose, $\mathrm{pH}$ 5.6; and $0.2 \mathrm{mM}$ Acetosyringone). The $\mathrm{OD}_{600}$ was measured with a spectrophotometer. The infiltration samples were mixed 1:1 at a total $\mathrm{OD}_{600}$ of 0.8. Samples were incubated at RT for 2 hours and infiltrated into the underside of Nicotiana benthamiana leaves with a $1 \mathrm{~mL}$ syringe. After two days, leaf samples were cut out with a razor blade and imaged with a confocal microscope.

Protoplast transfection and FLIM measurements were performed on a Leica SP8 as described 42 .

\section{Structural homology modeling}

SwissModel (https://swissmodel.expasy.org/) was used to model the structure of DIX-LIKE. To this end, the conserved N-terminal part of SOK1 was entered into the program and the software itself selected the best matching crystal structure, which was human Dvl2 (PBD: 4WIP).

\section{Statistics and image editing}

For all experiments involving transgenic lines, care was taken to analyze multiple independent transgenics, where lines were selected based on representing non-extreme characteristics. Only results representing the majority of observations are shown. All sample and observation numbers are listed in Extended Table 3.

Raw microscopy images were collected, and brightness was uniformly modified across the entire image. When images were to be directly compared, brightness modifications were performed in an identical manner. 


\section{Supplementary Material}

Refer to Web version on PubMed Central for supplementary material.

\section{Acknowledgments}

We thank Dominique Hagemans and Vivienne Mol for support with experiments, and Eva Benkova for supporting P.M.. This work was supported by the European Research Council under the European Union's Seventh Framework Programme (FP7/2007-2013) under REA grant agreement $\mathrm{n}^{\circ}$ [291734], and a Marie Curie Fellowships (contract 753138) to S.Y., by a European Research Council grant (ERC-StG CELLPATTERN; contract 281573), and ALW Open Competition grant (820.02.019) and an ALW-VIDI grant (864.06.012) from the Netherlands Organization for Scientific Research (NWO) to D.W., an ALW-VENI grant (863.21.010) from the Netherlands Organization for Scientific Research (NWO) to C.t.H., a grant (831.13.001) from the Netherlands Organization for Scientific Research (NWO) to M.v.D., and a FEBS long-term fellowship to P.M..

\section{References}

1. Butler MT, Wallingford JB. Planar cell polarity in development and disease. Nat Rev Mol Cell Biol. 2017; 18:375-388. [PubMed: 28293032]

2. Nakamura M, Kiefer CS, Grebe M. Planar polarity, tissue polarity and planar morphogenesis in plants. Curr Opin Plant Biol. 2012; 15:593-600. [PubMed: 22906885]

3. Johnston D, Ahringer J. Cell polarity in eggs and epithelia: Parallels and diversity. Cell. 2010; 141:757-774. [PubMed: 20510924]

4. Chiou J-G, Balasubramanian MK, Lew DJ. Cell Polarity in Yeast. Annu Rev Cell Dev Biol. 2017; 33:77-101. [PubMed: 28783960]

5. Johnston D. Establishing and transducing cell polarity: common themes and variations. Curr Opin Cell Biol. 2018; 51:33-41. [PubMed: 29153703]

6. Yoshida $\mathrm{S}$, et al. Genetic control of plant development by overriding a geometric division rule. Dev Cell. 2014; 29:75-87. [PubMed: 24684831]

7. De Rybel B, et al. A bHLH complex controls embryonic vascular tissue establishment and indeterminate growth in Arabidopsis. Dev Cell. 2013; 24:426-437. [PubMed: 23415953]

8. Gälweiler L, et al. Regulation of polar auxin transport by AtPIN1 in Arabidopsis vascular tissue. Science (80-. ). 1998; 282:2226-2230.

9. Müller A, et al. AtPIN2 defines a locus of Arabidopsis for root gravitropism control. EMBO J. 1998; 17:6903-6911. [PubMed: 9843496]

10. Friml J, Wiŝniewska J, Benková E, Mendgen K, Palme K. Lateral relocation of auxin efflux regulator PIN3 mediates tropism in Arabidopsis. Nature. 2002; 415:806-809. [PubMed: 11845211]

11. Friml J, et al. Efflux-dependent auxin gradients establish the apical-basal axis of Arabidopsis. Nature. 2003; 426:147-153. [PubMed: 14614497]

12. Langowski L, et al. Cellular mechanisms for cargo delivery and polarity maintenance at different polar domains in plant cells. Cell Discov. 2016; 2

13. Dong J, MacAlister CA, Bergmann DC. BASL Controls Asymmetric Cell Division in Arabidopsis. Cell. 2009; doi: 10.1016/j.cell.2009.04.018

14. Takano J, et al. Polar localization and degradation of Arabidopsis boron transporters through distinct trafficking pathways. Proc Natl Acad Sci. 2010; 107:5220-5225. [PubMed: 20194745]

15. Pillitteri LJ, Peterson KM, Horst RJ, Torii KU. Molecular profiling of stomatal meristemoids reveals new component of asymmetric cell division and commonalities among stem cell populations in Arabidopsis. Plant Cell. 2011; 23:3260-3275. [PubMed: 21963668]

16. Kania U, Fendrych M, Friml J. Polar delivery in plants; Commonalities and differences to animal epithelial cells. Open Biol. 2014; 4

17. Moller B, Weijers D. Auxin control of embryo patterning. Cold Spring Harb Perspect Biol. 2009; 1:a001545. [PubMed: 20066117] 
18. Hardtke CS, Berleth T. The Arabidopsis gene MONOPTEROS encodes a transcription factor mediating embryo axis formation and vascular development. EMBO J. 1998; 17:1405-1411. [PubMed: 9482737]

19. Berleth T, Jürgens G. The role of the monopteros gene in organising the basal body region of the Arabidopsis embryo. Development. 1993; 118:575-587.

20. Möller BK, et al. Auxin response cell-autonomously controls ground tissue initiation in the early Arabidopsis embryo. Proc Natl Acad Sci. 2017; 114:E2533-E2539. [PubMed: 28265057]

21. Schlereth A, et al. MONOPTEROS controls embryonic root initiation by regulating a mobile transcription factor. Nature. 2010; 464:913-916. [PubMed: 20220754]

22. Weijers D, et al. An Arabidopsis Minute-like phenotype caused by a semi-dominant mutation in a RIBOSOMAL PROTEIN S5 gene. Development. 2001; 128:4289-4299. [PubMed: 11684664]

23. Scheres B, et al. Mutations affecting the radial organisation of the Arabidopsis root display specific defects throughout the embryonic axis. Development. 1995; 121:53-62.

24. Benfey PN, et al. Root development in Arabidopsis: four mutants with dramatically altered root morphogenesis. Development. 1993; 119:57-70. [PubMed: 8275864]

25. Radoeva T, ten Hove CA, Saiga S, Weijers D. Molecular characterization of Arabidopsis GAL4/UAS enhancer trap lines identifies novel cell type-specific promoters. Plant Physiol. 2016; doi: $10.1104 / p p .16 .00213$

26. Alassimone J, Naseer S, Geldner N. A developmental framework for endodermal differentiation and polarity. Proc Natl Acad Sci. 2010; doi: 10.1073/pnas.0910772107

27. Liao CY, Weijers D. A toolkit for studying cellular reorganization during early embryogenesis in Arabidopsis thaliana. Plant J. 2018; 93:963-976. [PubMed: 29383853]

28. Traverso JA, et al. Roles of N-Terminal Fatty Acid Acylations in Membrane Compartment Partitioning: Arabidopsis h-Type Thioredoxins as a Case Study. Plant Cell. 2013; 25:1056-1077. [PubMed: 23543785]

29. Bienz M. Signalosome assembly by domains undergoing dynamic head-to-tail polymerization. Trends Biochem Sci. 2014; 10:487-495.

30. Ehebauer MT, Arias AM. The structural and functional determinants of the Axin and Dishevelled DIX domains. BMC Struct Biol. 2009; 9:70. [PubMed: 19909509]

31. Schwarz-Romond T, et al. The DIX domain of Dishevelled confers Wnt signaling by dynamic polymerization. Nat Struct Mol Biol. 2007; 14:484-492. [PubMed: 17529994]

32. Mansfield C, et al. Ectopic BASL Reveals Tissue Cell Polarity throughout Leaf Development in Arabidopsis thaliana. Curr Biol. 2018; 28:2638-2646. [PubMed: 30100337]

33. Radoeva T, ten Hove CA, Saiga S, Weijers D. Molecular characterization of Arabidopsis GAL4/UAS enhancer trap lines identifies novel cell type-specific promoters. Plant Physiol. 2016; doi: 10.1104/pp.16.00213

34. Fukaki H, et al. Genetic evidence that the endodermis is essential for shoot gravitropism in Arabidopsis thaliana. Plant J. 1998; 14:425-430. [PubMed: 9670559]

35. Helariutta Y, et al. The SHORT-ROOT gene controls radial patterning of the Arabidopsis root through radial signaling. Cell. 2000; 101:555-567. [PubMed: 10850497]

36. De Rybel B, et al. A versatile set of ligation-independent cloning vectors for functional studies in plants. Plant Physiol. 2011; 156:1292-1299. [PubMed: 21562332]

37. Weijers D, Van Hamburg JP, Van Rijn E, Hooykaas PJ, Offringa R. Diphtheria toxin-mediated cell ablation reveals interregional communication during Arabidopsis seed development. Plant Physiol. 2003; 133:1882-1892. [PubMed: 14605218]

38. Llavata-Peris C, Lokerse A, Moller B, De Rybel B, Weijers D. Imaging of phenotypes, gene expression, and protein localization during embryonic root formation in Arabidopsis. Methods Mol Biol. 2013; 959:137-148. [PubMed: 23299672]

39. Marhavý P, et al. Targeted cell elimination reveals an auxin-guided biphasic mode of lateral root initiation. Genes Dev. 2016; 30:471-483. [PubMed: 26883363]

40. von Wangenheim D, et al. Live tracking of moving samples in confocal microscopy for vertically grown roots. Elife. 2017; doi: 10.7554/eLife.26792 
41. de Reuille PB, et al. MorphoGraphX: A platform for quantifying morphogenesis in 4D. Elife. 2015; doi: 10.7554/eLife.05864

42. Rios AF, Radoeva T, Rybel B De, Weijers D, Borst JW. FRET-FLIM for Visualizing and Quantifying Protein Interactions in Live Plant Cells. Methods Mol Biol. 2017; 1497:135-146. [PubMed: 27864764] 

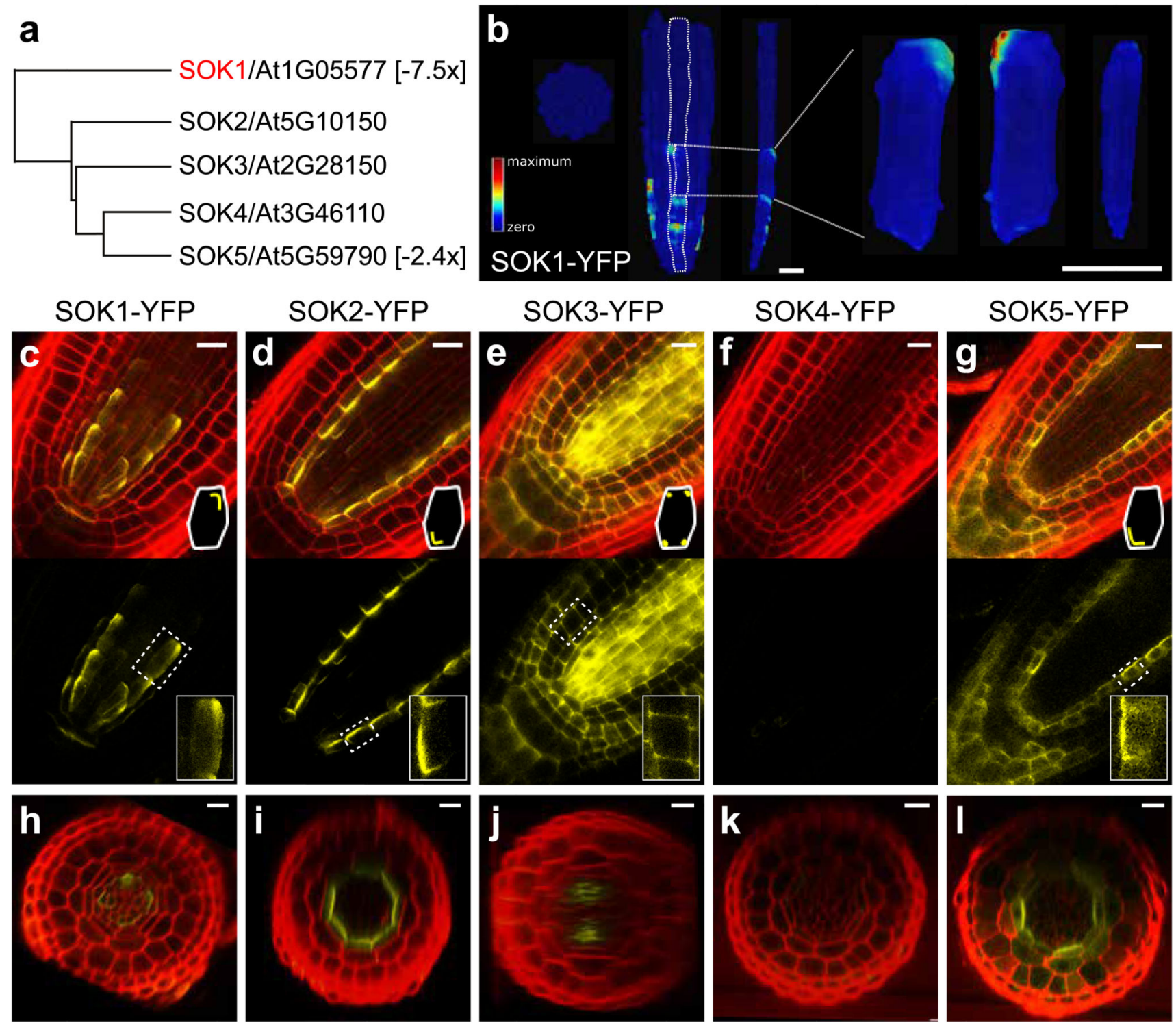

Fig 1. The SOSEKI family of polarly localized proteins.

(a) Phylogenetic tree of the Arabidopsis DUF966/SOSEKI protein family. Values in brackets indicate fold-change (downregulation) in Q0990>>bdl embryos20. (b) SOK1-YFP protein localization in a root tip. Fluorescence values are shown in false color (red=maximum; blue=zero) on segmented cell surfaces. From left to right: cross-section of vascular cylinder, surface view of vascular cylinder, single cell file, and 3 different views of a single segmented cell. (c-1) Localization of SOK1-YFP (c,h), SOK2-YFP (d,i), SOK3-YFP (e,j), SOK4-YFP $(\mathrm{f}, \mathrm{k})$ and SOK5-YFP $(\mathrm{g}, \mathrm{l})$ in longitudinal cross-sections (c-g) and transverse cross-sections (h-l) of primary root meristems counterstained with Propidium Iodide (red). Insets in c,d,e,g schematically show subcellular SOK protein localization. Bars $10 \mu \mathrm{m}$. All sample and observation numbers are listed in Extended Table 3. 

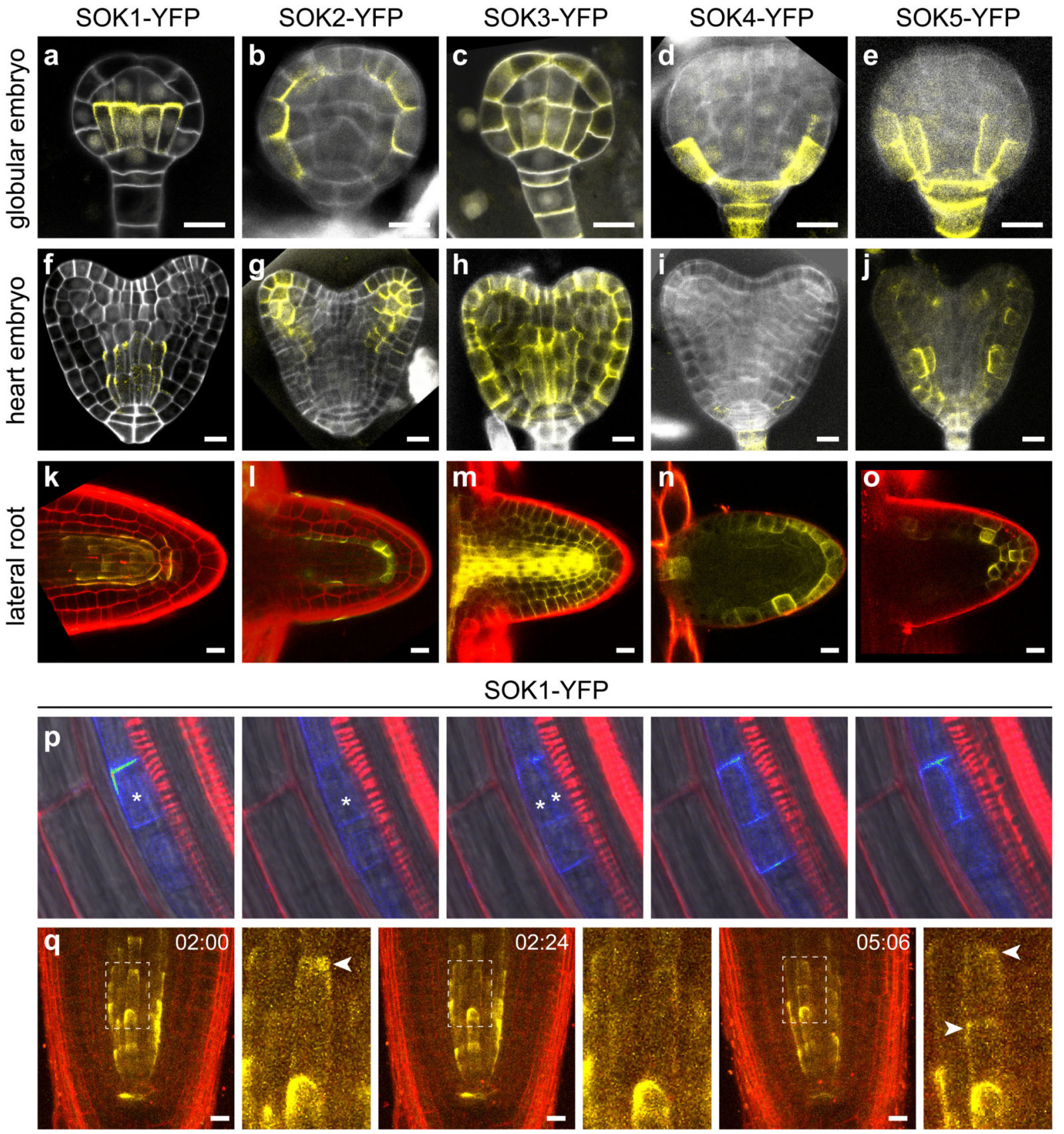

Fig 2. Diverse polar patterns of SOSEKI proteins

Localization of SOK1-YFP (a,f,k), SOK2-YFP (b,g,1), SOK3-YFP (c,h,m), SOK4-YFP

$(d, i, n)$ and SOK5-YFP (e,j,o) in globular stage embryos (a-e), heart stage embryos (f-j) and emerged lateral root primordia $(\mathrm{k}-\mathrm{o})$. $(\mathrm{p}, \mathrm{q})$ Stills of a time-lapse imaging series of SOK1YFP fluorescence in an initiating lateral root (p) and in the primary root tip (q). Time (in hours) is indicated in each panel and dividing cell lineages are marked by asterisks in (p). In (q), a dividing cell is magnified from the region indivated by a dashed box, and SOK1-YFP signal is indicated by arrowheads. Embryos in a-j are counterstained with Renaissance 
RS2200 (white), and roots (k-q) with Propidium Iodide (red). Bars $10 \mu \mathrm{m}$. All sample and observation numbers are listed in Extended Table 3. 

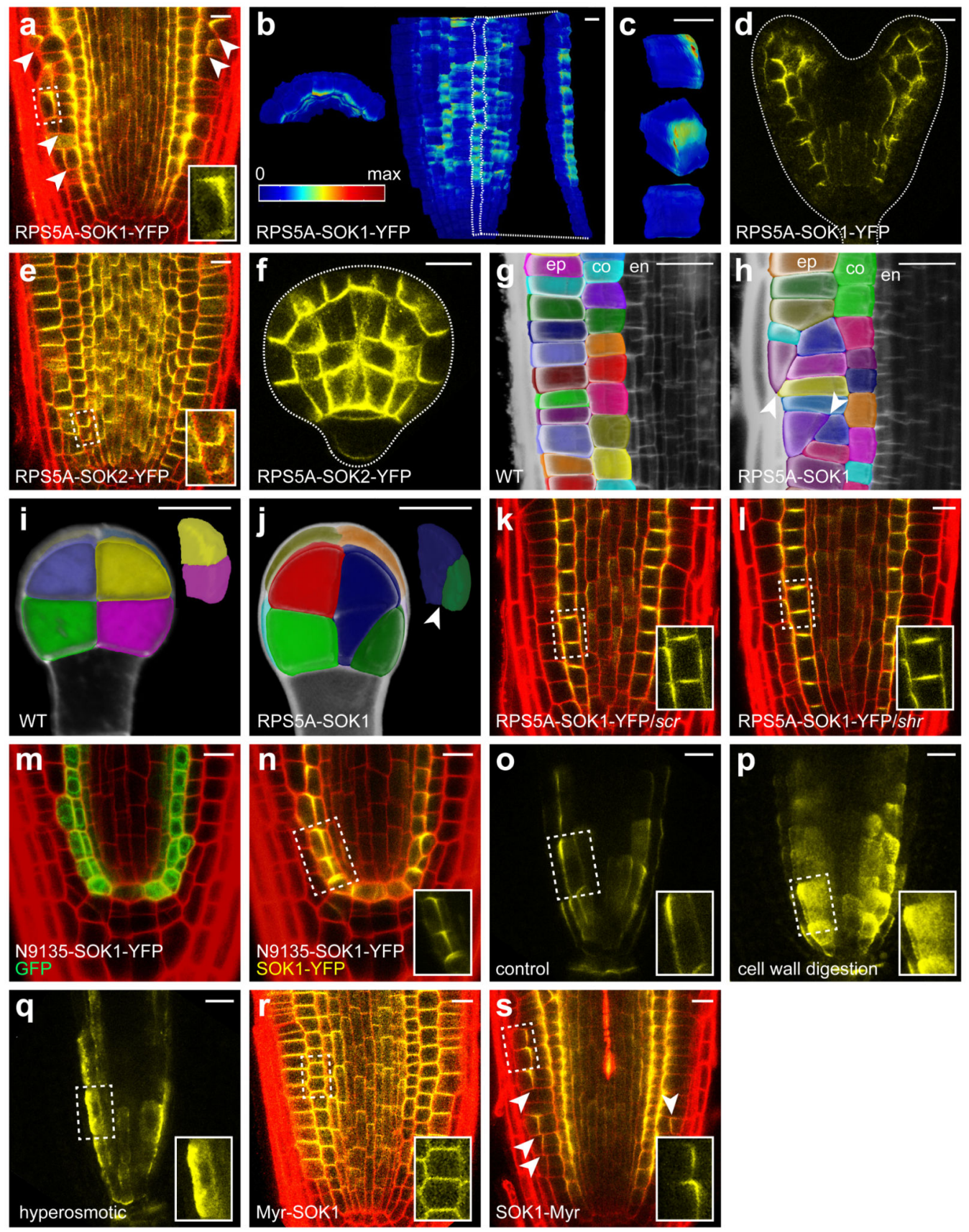

Fig 3. Regulation of SOSEKI localization

(a) Localization of SOK1-YFP in RPS5A-SOK1-YFP root tip. Arrowheads indicate examples of oblique cell divisions. (b,c) SOK1-YFP protein localization in a RPS5A-SOK1YFP root tip (b) and 3D localization of SOK1-YFP on segmented epidermal cells (c).

Fluorescence values are shown in false color (red=maximum; blue=zero) on segmented cell surfaces. (d) SOK1-YFP localization in RPS5A-SOK1-YFP heart stage embryo. (e,f) Localization of SOK2-YFP in RPS5A-SOK2-YFP root tip (e) and globular stage embryo (f). (g-j) Segmented cell volumes in wild-type (g,i) and RPS5A-SOK1 $(\mathrm{h}, \mathrm{j})$ root meristem (cells 
located between 70-160 $\mu \mathrm{m}$ away from the quiescent center were observed in g,h) and 8-cell embryo (i,j). (k,l) Localization of RPS5A-driven SOK1-YFP in $\operatorname{scr}((\mathrm{k})$ and $\operatorname{shr}(\mathrm{l})$ root tips. $(\mathrm{m}, \mathrm{n})$ GAL4-driven GFP expression (m) and GAL4-driven SOK1-YFP accumulation (n) in root tip of N9135 >>SOK1-YFP. (o-q) Localization of SOK1-YFP in control-treated root tip (o), and in root tips treated with cell wall-digesting enzymes for $3 \mathrm{~min}(\mathrm{p})$ or with $0.4 \mathrm{M}$ mannitol for $1.5 \mathrm{~h}$ (q). (r) Myr-SOK1-YFP localization in RPS5A- Myr-SOK1-YFP root tip. (s) SOK1-Myr-YFP localization in RPS5A-SOK1-Myr-YFP root tip. Arrowheads indicate oblique cell divisions. Walls in (a,e,g-n,r,s) are counterstained with Propidium Iodide (red). Insets in $(\mathrm{a}, \mathrm{e}, \mathrm{k}, \mathrm{l}, \mathrm{n}-\mathrm{s})$ show SOK protein localization in single cells corresponding to the area in dashed box. Bars $10 \mu \mathrm{m}$. All sample and observation numbers are listed in Extended Table 3. 
a
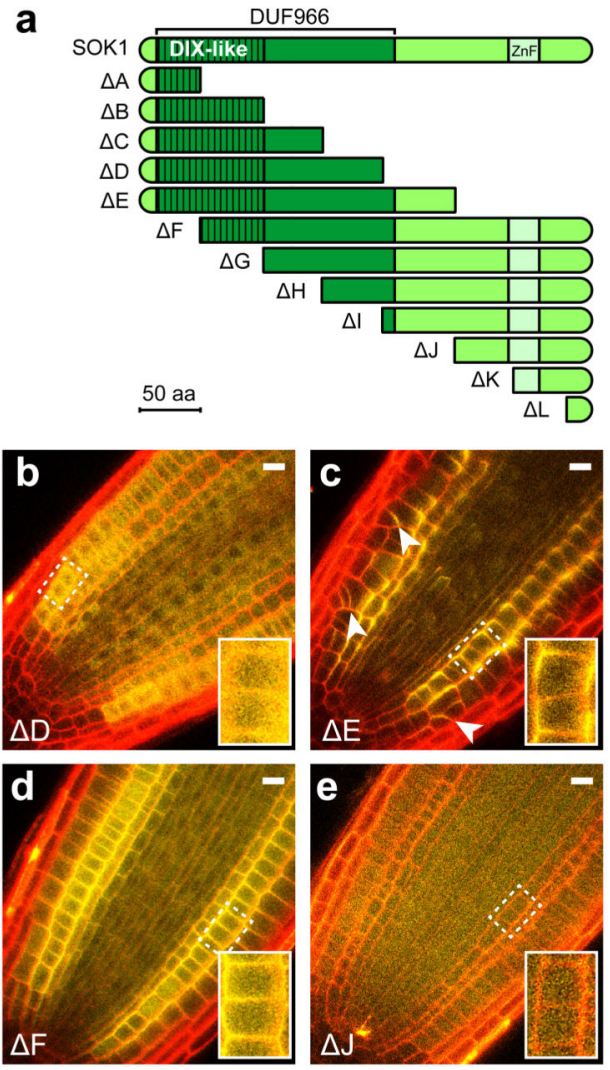

k

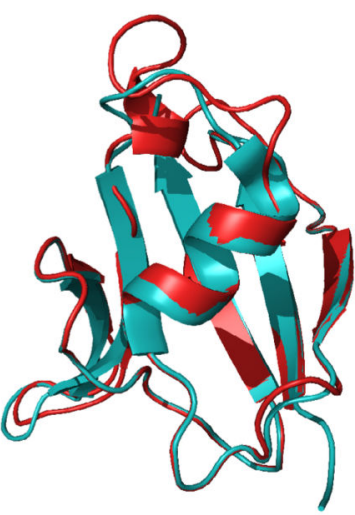

red: Arabidopsis SOK1 DIX-like cyan: human DvI2 DIX (4WIP) f

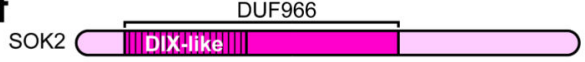

S2S1A $\quad$ (1)
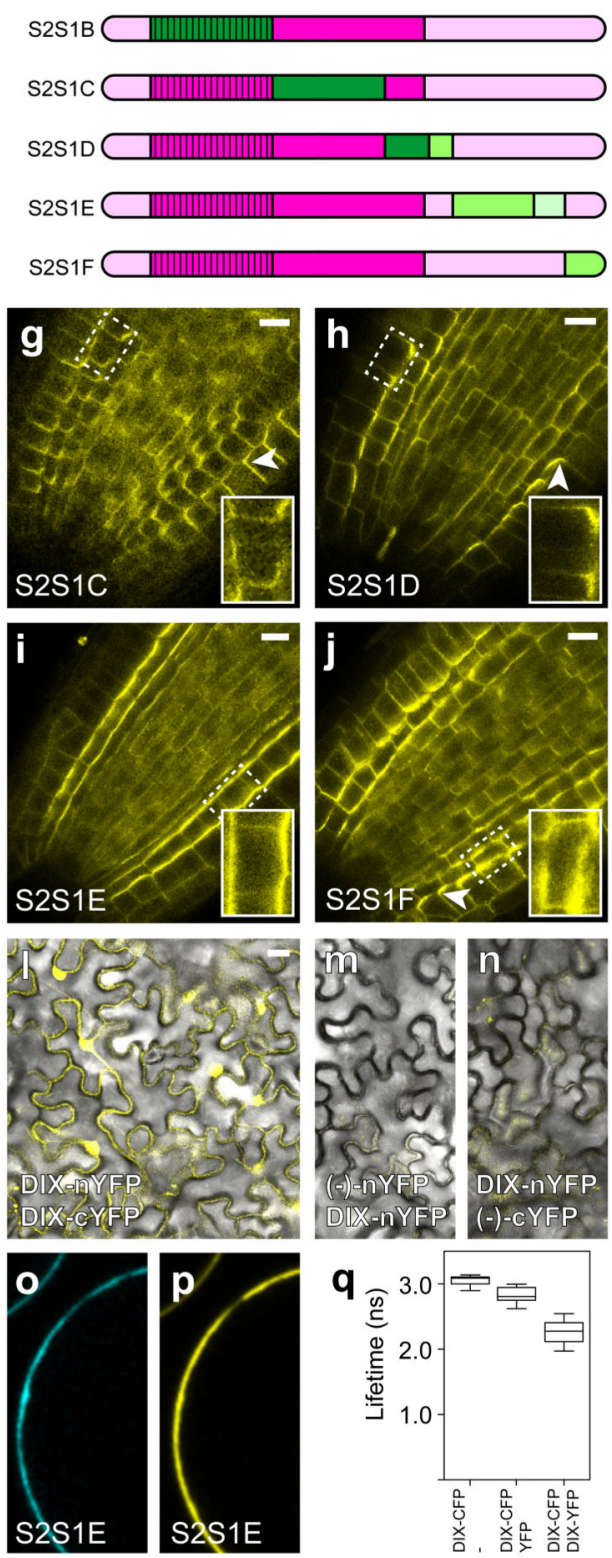

Figure 4. Protein determinants for SOK localization and activity

(a) Schematic of SOK1 protein domains ( $\mathrm{ZnF}=\mathrm{Zn}$-finger), and outline of domain deletion constructs expressed as YFP-fusions driven from the RPS5A promoter. (b-e) Representative examples of SOK1-YFP domain deletion accumulation in root tips. Arrowheads in (c) indicate examples of oblique divisions. (f) Outline of SOK2-SOK1 domain swaps, expressed as YFP fusions driven from the RPS5A promoter. (g-j) Representative examples of SOK1SOK2-YFP domain swap accumulation in root tips. Arrowheads in (g) and (j) mark basal localization, while arrowhead in (h) marks apical localization. (k) Structural alignment of the 
DIX domain in human DVL2 (PDB: 4WIP; blue) and the homology model of the SOK1 DIX-like domain (red). (1) Bimolecular Fluorescence complementation in Nicotiana benthamiana epidermis of the SOK1 DIX-like domain in homo-dimeric conformation, using C-terminal YFP fragments. $(m, n)$ BiFC control experiments with the DIX-like domain fused only to the n-terminal (m) or c-terminal (n) YFP half. $(o, p)$ Fluorescence of co-expressed SOK1 DIX-like domain as CFP (n) and YFP (o) fusion in protoplast. (q) Quantification of CFP Fluorescence Lifetime (in picoseconds, ps) in SOK1-DIX-CFP/YFP pair, and the two individual fusions in protoplasts. Boxplots represent values between upper and lower quartile, with median value shown, and error bars representing standard deviation. Walls in (b-e) are counterstained with Propidium Iodide (red). Bars $10 \mu \mathrm{m}$. Insets in (b-e,g-j) show SOK protein localization in single cells corresponding to the area in dashed box. Bars 10 $\mu \mathrm{m}$. All sample and observation numbers are listed in Extended Table 3. 